Natural Products as a Source for Novel Antibiotics

Mark G. Moloney*

Department of Chemistry, Chemistry Research Laboratory, The University of Oxford, 12 Mansfield Road, Oxford. OX1 3TA.

e-mail: mark.moloney@chem.ox.ac.uk

Abstract: Natural products have historically been of critical importance in the identification and development of therapeutically viable antibacterial agents. However, interest in these systems has waned in recent years, but the rapid emergence of resistant bacterial strains has forced their reevaluation as a route to identify novel chemical skeletons with antibacterial activity for elaboration in drug development. This overview examines the current situation, highlights new natural product systems which have been found, along with re-examination of some old ones, and new technologies for their identification. While natural products certainly have the potential to re-emerge once again as a key start point in antibacterial drug discovery, reports of new or reinvestigated structures need to be supported with sufficient quality chemical (solubility, stability), biochemical (including toxicity in particular, along with target information) and microbiological (MIC and resistance frequency) validation data to assist in the identification of genuinely promising hit structures, and to avoid wasted effort being expended by trawling over already cultivated territory. This is particularly important in a resource limited research environment.

Keywords: natural products; antibiotics; drug discovery

\title{
The Need for new antibiotics
}

The emergence of antimicrobial resistance (AMR), which began very soon after the introduction of antibiotics but has accelerated markedly over the last decade, has focused attention on the sustainability of current modern medical practice in the global arena.[1-3] From the perspective of an individual patient or an institutional health provider, assumptions - which have taken root over the last 70 years or so - about the nature, availability and outcomes of hitherto successful treatment protocols are being challenged, as community and hospital-acquired infections become more problematic.[4] The very success of antimicrobial drug discovery and therapies developed in the modern era has fostered a level of complacency which is likely to require substantial revision of medical practice in the coming decades, if we wish to maintain the standards of health care to which we have become accustomed, or to improve healthcare in underdeveloped countries.[5] Warnings of doomsday scenarios, perhaps dismissed all too readily in recent years, are beginning to look ever 
more plausible, especially with the recent emergence of colistin-resistant bacteria in China and even in the United Kingdom.[6, 7] Fortunately, the urgency of the task is now becoming more widely known and accepted, and has attracted the attention of governments and policymakers,[8-18] and led to the establishment of action groups (Antibiotic Action) [19] and charities (Antibacterial Research UK, ANTRUK).[20] That a concerted global approach is required, and that this will need to be an ongoing effort since all cells are capable of developing resistance to xenobiotics, has been recognised.[21, 22] A particular focus of current attention are the ESKAPE pathogens (Enterococcus faecium, Staphylococcus aureus, Klebsiella pneumoniae, Acinetobacter baumanii, Pseudomonas aeruginosa, and Enterobacter species) which are both responsible for a wide range of infections and increasingly resistant to common antibacterial drugs.[23] Application of antibiotics in agriculture and farming exacerbates environmental dispersal, and the implication for human use has been considered.[24]

The emergence of AMR has coincided with, or perhaps in part been caused by, an unfortunate series of circumstances. Firstly, the antibacterial drug pipeline is currently very poorly populated.[25-27] Secondly, commercially-driven models for drug development within the pharmaceutical industry, so successful during the last century, are looking very fragile for new antibacterial drug development in the twenty-first century.[12] Thirdly, restructuring within the industry has seen the loss of financial and human resource which translates to a serious diminution of drug-discovery capability.[28-31]. Fourthly, it now appears that there are challenges relating to bacterial cell wall ingress and egress which are unique to antibacterial drug discovery, and which significantly negatively impact on the probability of success.[32-37]. Although changes in the financial structure which supports antibacterial drug development and use are surely needed,[38] it nonetheless remains a productive activity, and antibacterials still comprise the biggest share of the global drug portfolio.[39] However, there is no doubt that high levels of collaboration will be required in order to address this problem, and an interesting case study of an effective academicindustrial partnership leading to antibacterial drug discovery highlights that a combination of chance, determination and adequate funding can generate material outcomes.[40] However, a recent note of caution, highlighting the need for adequate multidisciplinary training, in the way this should be done has been emphasised if academic laboratories are to make a valuable contribution in this endeavour, and not generate misleading outcomes.[41, 42]

An underpinning ethos critical to the success of modern antibacterial therapy has been the concept of the "magic bullet" - that is, a small molecule drug which is both selectively lethal for bacteria, and capable of mammalian administration. Alternative non-compound approaches (such as antibody, probiotic, phage and vaccine therapies) have recently been considered in detail, and their potential assessed.[43] Assuming that we adhere to the small molecule approach, a key 
question arises: how do we go forward to identify new drugs? Over the last century, natural products have provided a critical start point in antibacterial therapies and drug discovery[44] and because antibacterially active natural products appear to occupy an unusual chemical property space,[32, 45-47] this strategy still offers excellent opportunities for exploitation in drug development.[48] This realisation has led to a renaissance of interest in natural products for the identification of new members of the 'antibiotic-ome' (defined as natural products with antibiotic activity) and their application in antibacterial drug discovery in the genomics era.[40, 44, 45, 49-64] and that this need not necessarily result from random screening has been demonstrated very recently by the establishment of a relationship between protein-targeted or riboprotein-targeted antibacterial agents and their physicochemical properties.[45] This review attempts to show that recent developments in the natural products arena continue apace, and that reasons to expand work in this area are even greater under the current circumstances. In modern parlance, such an approach is referred to as "forward genetics," “forward pharmacology," "classical pharmacology” or "phenotypic drug discovery"[65] and there is an increasing realisation that it need not rely completely on serendipity, but that design rules may be identified,[66, 67] and some time ago an overview of the various databases listing natural products has appeared.[68] Whilst natural product isolation is often criticised for the fact that it only re-isolates known substances, and indeed this a serious disadvantage of this approach for the discovery of novel antibiotics, novel chemotypes continue to be discovered.[44, 69, 70] Natural sources of new antimicrobials and resistancemodifying agents include land plants,[71-73] fungi,[74] lichens,[75] endophytes,[76] as well as marine plants,[77-80] seaweeds,[81], corals,[82] and other marine microorganisms.[83, 84] A high throughput screening approach for the discovery of natural product antibiotics has recently been reported,[85] while cheminformatic approaches[86] and enhanced methodology for the identification of antibiotics[87] and producing strains[88] have also been developed. Of great significance is the development of novel genomic-type approaches to find small molecules with antibacterial activity, and it is likely that this will become an important avenue for the identification of novel opportunities in the future. [89-92] The particular value of natural products for the disruption of bacterial biofilms has been recognised[93-95] and so to has their antibacterial role in food[96, 97] textiles[98] and caries prevention.[99, 100]

\section{Opportunities for Natural Products in Antibiotic Drug Discovery}

This short review exemplifies the status of natural products with antibacterial activity by focusing on reports drawn largely from the last two years; the compound coverage is not meant to be comprehensive but rather illustrative. Noteworthy, though, is the breadth of chemical structures with wide-ranging antibacterial activity. Perhaps the single most important discovery in recent 
years has been that of the structurally unusual depsipeptide, teixobactin, which has generated a high degree of excitement, both for its intrinsic antibacterial activity, but also for its method of identification which involved culture of the producing bacterium in its natural soil environment.[101] New analogues have even been synthesised and evaluated in the short time since the original isolation was reported.[102] Importantly, teixobactin was found to inhibit cell wall synthesis by binding at both lipid II and lipid III, to possess MIC activity against a wide panel of Gram-positive organisms, to remain potent in the presence of blood serum, and to exhibit both microsomal stability and low toxicity.[101] Moreover, it was effective in vivo (S. aureus and Streptococcus pneumonia models). Other cyclic depsipeptides have been reported; examples are ulleungamides A and B with limited antibacterial activity (against S. aureus and Salmonella typhimurium) but no cytotoxicity; [103] salinamide F with significant Gram-positive and -negative activity (and potent activity against bacterial RNAP)[104] and further members of the enniatin class.[105] Copsin, isolated from a co-cultivated fungal source, is an antibiotic peptide which is unusually stabilized by six cysteine bonds, and which exhibits good activity for Gram-positive bacteria (B. subtilis, Listeria spp., and Enterococcus spp., and including vancomycin resistant $E$. faecium) but not Gram-negative species.[106] Copsin also inhibited cell wall biosynthesis by inhibition at lipid II. The structure of albicidin has been established, and is an unusual polyaromatic oligopeptide mainly composed of substituted $p$-aminobenzoic acids and linked by the nonproteinogenic $\alpha$-amino acid $\beta$-cyano-L-alanine.[107] It is a potent inhibitor of bacterial DNA gyrase (topoisomerase II) with excellent activity against a wide range of Gram-positive and negative bacteria. Cystobactamids are a similar system, but instead the $p$-aminobenzoamides are linked through an iso- $\beta$-methoxyasparagine or a $\beta$-methoxyasparagine unit. These too are bacterial DNA gyrase (topoisomerase II) inhibitors, and are effective against E. coli, A. baumannii, E. faecalis, S aureus and S. pneumonia.[108]

\section{Development of Small Molecules}

Small - and not so small - molecules continue to be found (Figure 1). Tetramatecontaining natural products[109] with potent antibacterial activity, include hymenosetin 1, only recently reported[110] and which has already succumbed to total synthesis.[111] Kibdelomycin 2 has been reported to exhibit broad-spectrum bactericidal activity, including against C. difficile, and to act by inhibiting DNA gyrase and topoisomerase IV;[112, 113] kibdelomycin A, a desmethyl analogue, has also been isolated.[114] Interestingly the dietziamides, tetramate dimers, were shown to possess antioxidative activity, but this did not translate into phenotypic antibacterial activity.[115] Hunanamycin A 3, containing a quinoxaline-2,3-dione core, was isolated from a marine-derived bacterium, $B$. hunanensis, and found to be selectively active against the bacterial 
pathogen Salmonella enterica and is postulated to be an inhibitor of riboflavin synthase.[70] Penicyclones A-E 4, antibacterial polyketides with a highly functionalized cyclohexenone core, were isolated from a Deep-Sea Penicillium species, and shown to have high levels of antimicrobial activity against the Gram-positive bacterium $S$. aureus, although were not found to be cytotoxic.[116]

Polyenic amides have been increasingly found which exhibit interesting antibacterial activity. Inthomycins A, B, and C 5a-c, all isomeric in the triene system, are known for their antibacterial activity, and have been prepared using highly efficient metal-catalysed coupling processes[117] which represent significant improvements over earlier work.[118] New simocyclinones (D9, D10, and D11), comprising aminocoumarin, polyketide and sugar sub-units linked by a tetraene, have been identified and this of interest since the parent, simocyclinone D8 6, is a DNA gyrase inhibitor with excellent activity against activity against both Gram-positive and negative bacteria.[119] Oxazolomycins 7a-d, known to possess limited antibacterial activity, comprise complex polyene lactam-lactone sub-structures and their total synthesis has been a focus of some considerable attention by Ishihara; their efforts open the door to more detailed microbiological examination of this interesting compound.[120] Batumin $\mathbf{8}$ is a general aminoacyl tRNA synthase inhibitor; it was also found to inhibit bacterial cell motility, although does not kill, and it has been suggested that it is not likely to induce resistance.[121] In that regard, it appears to be superior to the closely related system, mupirocin.

Other structurally unusual examples have been reported. The novel antibiotics, baulamycins A and B 9, capable of inhibiting growth of a range of both Gram-positive and -negative species, have been reported; these are inhibitors of a siderophore synthetase and their bacterial inhibitory activity against several bacterial species suggests that such targets might be worthy of more detailed investigation. [122, 123] Artonin I 10 has been reported to be an efflux pump inhibitor, to inhibit multidrug resistance in $S$. aureus and, when used in combination with existing antibiotics, to lower their MIC values.[124] Such "helper" systems offer the possibility of extending the lifetime of existing clinically effective drugs.

The realisation of the importance of finding new antibacterial agents, or developing old ones, has re-invigorated total synthesis in the total synthesis of antibacterials. The first total synthesis of tetarimycin A $\mathbf{1 1}$ has been reported, and the route been used to generate a small set of analogues, some of which were shown to be MRSA and VRE active; the presence of a critical hydroxyl group was essential for antibacterial activity.[125] Detailed analysis of viridicatumtoxin 12, the parent of an unusual class of polyketides closely related to the tetracycline antibiotics, indicates excellent inhibitory activity against vancomycin-resistant Enterococci and methicillinresistant S. aureus.[126] Of interest is both its higher acid stability and bioactivity over the 
corresponding tetracycline antibiotics, although it does show some cytotoxicity. Other new polyketides (e.g. barceloneic acid C) have been isolated from Phoma species, but found to have only weak or no activity.[127] The total synthesis of hongoquercin B $\mathbf{1 3}$ has been efficiently achieved by a biomimetic cascade tetracyclization.[128]

Alkaloids continue to be identified with antibacterial activity (Figure 2); detailed overviews of their biological (including antibacterial) activity have been reported.[129, 130] The development of a practical synthetic and fully scalable route to the axinellamines $\mathbf{1 4}$ has enabled a more detailed assessment of their antibacterial activity, which is favourable against both Gram-positive and negative bacteria; they appear to function by membrane destabilization.[131] Curvulamine 15, a structurally highly novel antibacterial alkaloid has been reported to be isolated from the white croaker, a fish observed to be resistant to infections which commonly affect other species,[130] and was found to be both more potent and selective than the positive control, tinidazole.

Combination therapies are a possible way forward, in which the synergistic action of complementary antibiotics is harnessed to achieve a better overall clinical outcome. It has been suggested that polyphenols, an intensively investigated and widely distributed group of natural products often possessing antibacterial activity, possess potential as therapeutic agents alone or in combination with currently available antibiotics[132] and recent developments in antibacterial activities of chalcones has been reviewed.[133] The co-administration of antibiotics, or their chemical conjugation, has been reported to lead to a better therapeutic outcome than their use individually. Synthesis of tridecaptin conjugated with any of several antibiotics (vancomycin, rifampicin, erythromycin A) using Click chemistry provided access to a novel library, and conjugation with the latter particularly gave a hybrid with excellent MIC against Klebsiella pneumoniae.[134] The synergistic activity of epigallocatechin gallate and quercetin when used in combination has been shown to be particularly effective against a topical methicillin-resistant $S$. aureus infection.[135]

\section{A Case Study - Antitubercular Agents}

A particular area in which natural product isolation and synthesis has remained both popular and productive is in the discovery of antitubercular agents,[136, 137] and while terpenoid[138] and coumarin[139] systems are well known, newer structures continue to be found (Figures 3 and 4). The application of more modern approaches has also proven to be valuable: a virtual screening protocol optimizing physicochemical and pharmacokinetic parameters against rhamnose pathway enzymes has been able to identify compounds with good binding affinity, including butein, diospyrin, indicanine, and rumexneposide A, and antitubercular activity was confirmed for butein.[140] By contrast, a return to whole-cell screening has enabled the identification of 
structurally diverse novel antitubercular hits.[141] Natural product isolation has yielded diverse hits with promising activity. These include two new marine diterpenoids $\mathbf{1 6 a}, \mathbf{b}$, belonging to the unusual isoneoamphilectane-class, isolated from the marine sponge Svenzea flava, along with semisynthetic derivative 16c and other known amphilectane diterpenes, which exhibited both strong growth inhibition of Mycobacterium tuberculosis H37Rv and low mammalian toxicity.[142] Complex tetronates lobophorins A, B and G were isolated from a newly isolated Actinomycetes strain using a bioassay-guided isolation protocol, and shown to possess moderate anti-M. tuberculosis H37Rv activity but strong inhibition against against B. subtilis.[143] Alkaloids, too, continue to be found, and include denigrins A-C 17a-c, new 3,4-diarylpyrrole alkaloids from the marine sponge Dendrilla nigra,[144] again identified by bioactivity guided isolation protocol, with denigrin C offering the most activity against M. tuberculosis H37Rv. Phomapyrrolidones A-C 18ac, containing both a cyclopenta[b]fluorene and succinimide ring system, were isolated from the endophytic Phoma sp. fungus, but exhibited only weak antitubercular activity below their cytotoxic concentrations, with phomapyrrolidone $C$ being the most active.[145] Caulerpin 19 and some closely related analogues $19(\mathrm{R}=\mathrm{F}, \mathrm{Cl}, \mathrm{Br}, \mathrm{I}, \mathrm{Me}, \mathrm{OMe})$, all bis-indole alkaloids, were synthesized in a short efficient cyclocondensation sequence, but only the parent natural product exhibited significant anti-tuberculosis activity.[146] Agelasines B, C and D 20a-c, known antituberculars, were reisolated from an Indonesian marine sponge of the genus Agelas, and agelasine D were found to bind to the target protein BCG3185c $\left(\mathrm{K}_{\mathrm{D}} 2.42 \mu \mathrm{M}\right)$, and to have the best activity against $M$. smegmatis and M. bovis BCG under aerobic and hypoxic conditions.[147] Quinonoid systems regularly crop up: four new pluramycins, heraclemycins A-D 21a-d, were isolated from an endophytic Streptomyces sp which have been used in traditional Chinese medicine, with heraclemycin C in particular showing selective antitubercular activity, while heraclemycin D was active against $S$. aureus, Methicillin-resistant S. aureus, and B. subtilis but not Pseudomonas aeruginosa.[148] New natural products, taiwanensols A, B and C 22a-c, were isolated using a bioassay-guided protocol and found to possess some antitubercular activity.[149] The known 5-(3-hydroxyphenyl)-penta-2,4dienamide, ergosterol, ergosterol peroxide and halolitoralin B were all recently isolated from an endophytic Streptomyces sp, and all shown to exhibit anti-M. Tuberculosis activity.[150]

The synthetic and biological re-evaluation of known natural products is also gaining attention (Figure 4). A wide variety of natural products were screened for activity against $M$. tuberculosis, and of these, vermelhotin 23, a tetramic acid isolated from fungi, was found to be the most active.[151] Further investigation of the natural products emodin, diospyrin, plumbagin, menadione and thymoquinone were evaluated for antitubercular activity, and shown to be active, with the best compound, plumbagin, reported to be effective at $0.25 \mu \mathrm{g} / \mathrm{mL}$.[152] 
Synthetic and semi-synthetic approaches have also proven to be effective in the identification of new antitubercular actives. For example, a natural product inspired application of Ugi four-component couplings of amines, aldehydes, acids with a diisocyanide to generate antitubercular and antimalarial pharmacophores has been shown to give $\alpha$-acylamino amides 24 with good anti-infective activity and selectivity, and were found also to inhibit in vitro thromboxane B2 (TXB2) and superoxide anion generation.[153] Similarly, mimics of the natural product engelhardione (published structure) $\mathbf{2 5}$ and its revised structure (pterocarine) $\mathbf{2 6}$ have been prepared using a short sequence, and some were shown to have Mycobacterium tuberculosis along with more general Gram-positive and -negative activity.[154] Commercially available oridonin 27 has been modified in a series of transformations to introduce a range of functional groups onto the core tetracyclic skeleton; evaluation of their antimycobacterial activity indicated some activity, and most importantly, against M. tuberculosis H37Rv.[155, 156] Modified griselimycins, all cyclic peptides, exhibit excellent MIC values with improved metabolic stability and hydrophobicity, leading to improved cell membrane permeability.[157] In a different approach, biotransformations of monoterpenoids has been used to generate antibacterial actives.[158] Improvements in the total synthesis of a number of natural products has opened the door for their further investigation. This includes the enantioselective total synthesis of thuggacin B 28[159] (active against M. tuberculosis with a novel mode of action) and hirsutellones A,B and C 29 (strongly active against a resistant strain of M.tuberculosis).[160] Caprazamycin is a complex nucleoside derivative which incorporates a number of key structural elements, including a diazepanone ring, an amino ribose, a pyranose, a uridine, and a fatty-acid side chain with activity against against Mycobacterium tuberculosis (TB), including multidrug-resistant variants. Its first total synthesis, achieved in 23 steps, has been reported and is noteworthy because it is scalable and opens the possibility of more detailed examination of SAR of this class of antibiotics.[161] An effective route to the simpler analogue capuramycin 30, and its analog UT-01309, using elegantly designed new protecting groups specifically designed for the primary alcohol and uridine ureido nitrogen groups of the target, and which permits the synthesis of gram-quantities of an advanced intermediate suitable for elaboration to capuramycin and its analogs in as few as four steps, has been reported.[162]

\section{Concluding Remarks}

Although a recent study has identified the opportunities which might arise from non-small molecule antibacterial therapies,[43] it is clear that there is ample scope for innovation inspired by an understanding of natural product chemistry, and many have argued that this approach should be re-activated as a matter of some urgency.[31, 163-167] Indeed there is still a danger that the importance of serendipitously discovered small molecules will be de-emphasised relative to 
“rational” target driven approaches. A recent analysis of drugs approved by the USA FDA drugs has again come to the conclusion both that natural products and their derivatives have both played a key role in the past and are likely to be a productive way forward in the future, and that appropriate investment from private and public sectors is justified.[168] Certainly, small molecules, and many of them related to natural products, offer interesting opportunities for the identification of short term solutions using resistance reversal agents[124, 169] and resistance breakers; an example is artonin 10 (vide supra).[170] Notwithstanding the opportunities for the discovery of new natural products (perhaps using new approaches) outlined above, there is nonetheless plenty of life left in these known systems too, if they are examined in detail; this provides a different possible approach to the development of short term solutions by re-invigorating historically effective antibacterial small molecules. Critically, it is wrong to assume that well known and currently clinically used systems have been optimised fully during their development, and in any case historical optimisation was not made against parameters relevant in the modern context. A good example is the recent identification of next-generation polymixin lipopeptides $\mathbf{3 0}$ with both superior activity against MDR Gram-negatives and superior pharmacokinetic profiles when compared to colistin; this was achieved by a "classical” SAR study in which three key components of the polymixin framework were systematically modified and evaluated in detail.[171, 172] Other examples include tetarimycin 11 and the viridicatumtoxins 12 (vide supra), the latter of which are fungal polyketides which are closely related to the tetracycline antibiotics but which have not found clinical application.[126] Their isolation and detailed structural assignment followed by antibacterial and cytotoxic characterisation identified promising activity, but importantly also that the viridicatumtoxin scaffold was significantly more acid-stable than the tetracycline antibiotics themselves, and opening the door to the development of new next-generation systems in this important class of antibiotics. In a slightly different approach, the well known antibacterial activity of tetramate-containing natural products,[173, 174] exemplified by streptolydigin 31, has inspired the construction of small molecule analogues which have been found to exhibit promising levels of antibacterial activity.[175-177] The very fact that many antibacterial active natural products are comprised of clearly define structural subunits - arising of course from the metabolic process of cellular biosynthetic machinery - provides an easy opportunity for medicinal chemists to rapidly and effectively create mimics for optimisation for biological parameters.

But if natural products chemistry is to make an impact in the development of new antibacterial drugs, and especially in an academic context, reports of new or reinvestigated structures need to be supported with sufficient quality chemical (solubility, stability), biochemical (including toxicity in particular, along with target information) and microbiological (MIC and resistance frequency) data which permits the scientific community to assess if the system is worthy 
of further examination - and many of the reports included here show the value of inclusion of that information. However, natural products are not immune to providing false leads, and the importance of the recognition of, and appropriate response to, pan-assay interference has recently been highlighted, and is something to which academic groups need to pay particular attention.[178, 179] Fortunately, acquiring this data is now much more readily possible with the availability of a world-wide open access phenotypic screening service, CO-ADD,[180, 181] which embraces the crowd sourcing model for drug discovery. Moreover, a greater understanding of the 'antibioticome', its relationship to natural products, and methods for identifying their modes of action, is likely to provide greater predictive power for the identification of worthwhile avenues to explore[52, 182] and this type of approach is finding commercial application.[183] In the race to find new antibacterials, there is good reason to be optimistic, but diverse strategies will need to be adequately resourced and there is no room for complacency!

\section{References}

1 Anderson, A.C., et al. (2011) The challenge of developing robust drugs to overcome resistance. Drug Discovery Today 16, 755-761

2 Hurdle, J.G., et al. (2011) Targeting bacterial membrane function: an underexploited mechanism for treating persistent infections. Nat. Rev. Microbiol. 9, 62-75

3 Wright, G.D. (2011) Molecular mechanisms of antibiotic resistance. Chem. Commun. 47, 40554061

4 Mulvey, M.R. and Simor, A.E. (2009) Antimicrobial resistance in hospitals: How concerned should we be? Can. Med. Assoc. J. 180, 408-415

5 Perros, M. (2015) A sustainable model for antibiotics. Science 347, 1062-1064

6 Baker, S. (2015) A return to the pre-antimicrobial era? Science 347, 1064-1066

7 Liu, Y.-Y., et al. (2015) Emergence of plasmid-mediated colistin resistance mechanism MCR-1 in animals and human beings in China: a microbiological and molecular biological study. Lancet Infect. Dis. 16, 161-168

8 (2015) Responding to the threat of antimicrobial resistance: Australia's First National Antimicrobial Resistance Strategy 2015-2019, Departments of Health and Agriculture, Australian Government, June 2015.

<http://www.health.gov.au/internet/main/publishing.nsf/Content/1803C433C71415CACA257C840 0121B1F/\$File/amr-strategy-2015-2019.pdf.

9 (2015) Federal action plan on antimicrobial resistance and use in Canada, Public Health Agency of Canada, March 2015. < http://healthycanadians.gc.ca/alt/pdf/publications/drugs-productsmedicaments-produits/antibiotic-resistance-antibiotique/action-plan-daction-eng.pdf $>$ 10 (2015) National Action Plan for combating antibiotic-resistant bacteria, The White House, Washington, March 2015 <https://www.whitehouse.gov/sites/.../pcast_carb_report_sept2014.pdf> 11 Gelband, H., et al. (2015) State of the World's Antibiotics, 2015. Center for Disease Dynamics, Economics \& Policy. 2015.

12 Kinch, M.S., et al. (2014) An analysis of FDA-approved drugs for infectious disease:

antibacterial agents. Drug Discovery Today 19, 1283-1287

13 O'Neill, J. (2014) Antimicrobial Resistance:Tackling a crisis for the health and wealth of nations (http://amr-review.org).

14 (2014) Antimicrobial Resistance. Global Report on Surveillance. World Health Organization 
15 (2014) Report to the President on combating antibiotic resistance, Sept 2014.

$<$ https://www.whitehouse.gov/sites/.../pcast_carb_report_sept2014.pdf $>$

16 (2013) U.S. Department of Health and Human Services (Centres for Disease Control and Prevention). http://www.cdc.gov/drugresistance/threat-report-2013/

17 (2013) UK Five Year Antimicrobial Resistance Strategy 2013-2018. Department for Health and Department for Environment, Food and Rural Affairs

18 Wise, R. and Piddock, L.J.V. (2010) The Urgent Need Regenerating antibacterial drug discovery development. British Society for Antimicrobial Chemotherapy

19 Antibiotic Action. http://antibiotic-action.com/

20 ANTRUK. http://www.antibioticresearch.org.uk/

21 Laxminarayan, R., et al. (2014) Antibiotic resistance - the need for global solutions. Lancet 13, 1057-1098

22 Howard, S.J., et al. (2013) Antibiotic resistance: global response needed. Lancet Infect. Dis. 13, 1001-1003

23 Tommasi, R., et al. (2015) ESKAPEing the labyrinth of antibacterial discovery Nat. Rev. Drug Discovery 14, 529

24 Wang, J., et al. (2015) Human-use antibacterial residues in the natural environment of China: implication for ecopharmacovigilance. Environmental Monitoring and Assessment 187, 331-331 25 Boucher, H.W., et al. (2013) $10 \times$ ’20 Progress—Development of New Drugs Active Against Gram-Negative Bacilli: An Update From the Infectious Diseases Society of America. Clin. Infect. Dis. 56, 1685-1694

26 Butler, M.S., et al. (2013) Antibiotics in the clinical pipeline in 2013. $J$ Antibiot 66, 571-591 27 Saleem, M., et al. (2010) Antimicrobial natural products: an update on future antibiotic drug candidates. Nat. Prod. Rep. 27, 238-254

28 Walsh, C.T. and Wencewicz, T.A. (2014) Prospects for new antibiotics: a molecule-centered perspective. J. Antibiot. 67, 7-22

29 Butera, J.A. (2013) Phenotypic Screening as a Strategic Component of Drug Discovery Programs Targeting Novel Antiparasitic and Antimycobacterial Agents. J. Med. Chem. 56, $7715-7718$

30 Zheng, W., et al. (2013) Phenotypic screens as a renewed approach for drug discovery. Drug Discov Today 18, 1067-1073

31 Newman, D.J. and Cragg, G.M. (2012) Natural Products As Sources of New Drugs over the 30 Years from 1981 to 2010. J. Nat. Prod. 75, 311-335

32 Singh, S.B. (2014) Confronting the challenges of discovery of novel antibacterial agents. Biorg. Med. Chem. Lett. 24, 3683-3689

33 Silver, L.L. (2011) Challenges of Antibacterial Discovery. Clin. Microbiol. Rev. 24, 71-109

34 Dick, T. and Young, D. (2011) How antibacterials really work: impact on drug discovery.

Future Microbiol. 6, 603-604

35 Gwynn, M.N., et al. (2010) Challenges of antibacterial discovery revisited. Ann. N.Y. Acad. Sci. 1213, 5-19

36 Williams, K.J. and Bax, R.P. (2009) Challenges in developing new Antibacterial Drugs. Curr. Opin. Invest. Drugs 10, 157-163

37 Payne, D.J., et al. (2007) Drugs for bad bugs: confronting the challenges of antibacterial discovery. Nat. Rev. Drug Discovery 6, 29-40

38 Hwang, T.J., et al. (2015) Paying for innovation: Reimbursement incentives for antibiotics. Sci. Transl. Med. 7, 276fs279-276fs279

39 Kinch, M.S., et al. (2014) Trends in pharmaceutical targeting of clinical indications: 1930-2013. Drug Discovery Today 19, 1682-1685

40 Mann, J., et al. (2015) The discovery of a novel antibiotic for the treatment of Clostridium difficile infections: a story of an effective academic-industrial partnership. Med. Chem. Commun. 6, 1420 
41 Baell, J.B. (2015) Screening-Based Translation of Public Research Encounters Painful Problems. ACS Med. Chem. Lett. 6, 229-234

42 Whitty, A. (2011) Growing Pains in Academic Drug Discovery. Future Med Chem. 3, 797-801

43 Czaplewski, L., et al. (2016) Alternatives to antibiotics; a pipeline portfolio review. Lancet Infect. Dis. 16, 239-251

44 Brown, D.G., et al. (2014) New natural products as new leads for antibacterial drug discovery. Biorg. Med. Chem. Lett. 24, 413-418

45 Mugumbate, G. and Overington, J.P. (2015) The relationship between target-class and the physicochemical properties of antibacterial drugs. Biorg. Med. Chem. 23, 5218-5224

46 O'Shea, R. and Moser, H.E. (2008) Physicochemical Properties of Antibacterial Compounds: Implications for Drug Discovery. J. Med. Chem. 51, 2871-2878

47 O’Neill, A.J. and Chopra, I. (2004) Preclinical evaluation of novel antibacterial agents by microbiological and molecular techniques. Expert Opin. Drug Discov. 13, 1045-1063

48 Quinn, R.J., et al. (2008) Developing a Drug-like Natural Product Library. J. Nat. Prod. 71, 464-446

49 Harvey, A.L., et al. (2015) The re emergence of natural products for drug discovery in the genomics era Nat. Rev. Drug Discovery 14, 111-129

50 Holban, A.M. and Grumezescu, A.M. (2015) Antimicrobial Strategies based on Natural

Products: Recent Progress in Bio and Nanotechnology. Curr. Pharm. Biotechnol. 16, 85-86

$51 \mathrm{Hu}$, Y., et al. (2015) Statistical Research on the Bioactivity of New Marine Natural Products

Discovered during the 28 Years from 1985 to 2012. Mar. Drugs 13, 202-221

52 Johnston, C.W. and Magarvey, N.A. (2015) Natural Products: Untwisting the antibiotic'ome.

Nat. Chem. Biol. 11, 177-178

53 Mogosanu, G.D., et al. (2015) Prevention of Microbial Communities: Novel Approaches Based

Natural Products. Curr. Pharm. Biotechnol. 16, 94-111

54 Bathula, S.R., et al. (2015) Pruning of biomolecules and natural products (PBNP): an innovative paradigm in drug discovery. Org. Biomol. Chem. 16, 6432

55 Brown, P. and Dawson, M.J. (2015) A perspective on the next generation of antibacterial agents derived by manipulation of natural products. Prog. Med. Chem. 54, 135-184

56 Idhayadhulla, A., et al. (2014) Synthesis of novel and diverse mollugin analogues and their antibacterial and antioxidant activities. Bioorg. Chem. 52, 77-82

57 Khan, F.A., et al. (2014) Syntheses of a library of molecules on the marine natural product ianthelliformisamines platform and their biological evaluation. Org. Biomol. Chem. 12, 3847-3865

58 Li, G., et al. (2014) Natural products containing 'decalin' motif in microorganisms. Nat. Prod.

Rep. 31, 1175-1201

59 Cragg, G. and Newman, D. (2014) Natural products and drug discovery and development: A history of success and continuing promise for the future. Planta Medica 80, 750-750

60 Dashti, Y., et al. (2014) Predicting natural product value, an exploration of anti-TB drug space.

Nat.l Prod. Rep. 31, 990-998

61 Genilloud, O. (2014) The re-emerging role of microbial natural products in antibiotic discovery.

Antonie Van Leeuwenhoek International Journal of General and Molecular Microbiology 106, 173188

62 Georgiev, M.I. (2014) Natural products utilization. Phytochem. Rev. 13, 339-341

63 Gyawali, R. and Ibrahim, S.A. (2014) Natural products as antimicrobial agents. Food Control 46, 412-429

64 Wright, G.D. (2014) Something old, something new: revisiting natural products in antibiotic

drug discovery. Can. J. Microbiol. 60, 147-154

65 Takenaka, T. (2001) Classical vs reverse pharmacology in drug discovery. BJU International 88(Suppl. 2), 7-10

66 Katsuno, K., et al. (2015) Hit and lead criteria in drug discovery for infectious diseases of the developing world. Nat. Rev. Drug Discovery 14, 751-758 
67 Camp, D., et al. (2012) Drug-like Properties: Guiding Principles for the Design of Natural Product Libraries. J. Nat. Prod. 75, 72-81

68 Fullbeck, M., et al. (2006) Natural products: sources and databases. Nat. Prod. Rep. 23, 347-356

69 Bonifacio, B.V., et al. (2014) Antimicrobial activity of natural products against Helicobacter pylori: a review. Ann. Clin. Microbiol. Antimicrob. 13, 54

$70 \mathrm{Hu}$, Y., et al. (2013) Hunanamycin A, an Antibiotic from a Marine-Derived Bacillus hunanensis.

Org. Lett. 15

71 Yong, A.-L., et al. (2015) Investigation of antibacterial mechanism and identification of bacterial protein targets mediated by antibacterial medicinal plant extracts. Food Chemistry 186, 32-36

72 Vandal, J., et al. (2015) Antimicrobial activity of natural products from the flora of Northern Ontario, Canada. Pharmaceutical Biology 53, 800-806

73 Gechev, T.S., et al. (2014) Natural products from resurrection plants: Potential for medical applications. Biotechnol. Adv. 32, 1091-1101

$74 \mathrm{Wu}, \mathrm{C}$., et al. (2015) Expanding the chemical space for natural products by Aspergillus-

Streptomyces co-cultivation and biotransformation. Scientific Reports 5

75 Yousuf, S., et al. (2014) Lichens: Chemistry and biological activities. In Studies in Natural

Products Chemistry, pp. 223-259

76 Deshmukh, S.K., et al. (2015) Endophytic fungi: a reservoir of antibacterials. Front. Microbiol. 5:715

77 Ng, T.B., et al. (2015) Antibacterial products of marine organisms. Appl. Microbiol. Biotechnol. 99, 4145-4173

78 Kiuru, P., et al. (2014) Exploring Marine Resources for Bioactive Compounds. Planta Medica 80, 1234-1246

79 Mondol, M.A.M. and Shin, H.J. (2014) Antibacterial and Antiyeast Compounds from MarineDerived Bacteria. Mar. Drugs 12, 2913-2921

80 Mayer, A.M.S., et al. (2013) Marine Pharmacology in 2009-2011: Marine Compounds with Antibacterial, Antidiabetic, Antifungal, Anti-Inflammatory, Antiprotozoal, Antituberculosis, and Antiviral Activities; Affecting the Immune and Nervous Systems, and other Miscellaneous Mechanisms of Action. Mar. Drugs 11, 2510-2573

81 Singh, R.P., et al. (2015) Antimicrobial compounds from seaweeds-associated bacteria and fungi. Appl. Microbiol. Biotechnol. 99, 1571-1586

82 Dobretsov, S., et al. (2015) Inhibition of bacterial fouling by soft coral natural products. Int. Biodeterior. Biodegrad. 98, 53-58

83 El Amraoui, B., et al. (2014) Antifungal and antibacterial activity of marine microorganisms. Ann. Pharm. Fr. 72, 107-111

84 Monciardini, P., et al. (2014) Discovering new bioactive molecules from microbial sources. Microbial Biotechnology 7, 209-220

85 Scanlon, T.C., et al. (2014) A High-Throughput Screen for Antibiotic Drug Discovery.

Biotechnology and Bioengineering 111, 232-243

86 Pereira, F., et al. (2014) A Chemoinformatics Approach to the Discovery of Lead-Like

Molecules from Marine and Microbial Sources En Route to Antitumor and Antibiotic Drugs. Mar.

Drugs 12, 757-778

87 Linington, R.G. (2014) Connecting phenotype and chemotype: Development of an integrated platform for the direct prediction of natural product modes of action. Planta Medica 80, 757-758 88 Thaker, M.N., et al. (2014) Antibiotic resistance-mediated isolation of scaffold-specific natural product producers. Nature Protocols 9, 1469-1479

89 Rutledge, P.J. and Challis, G.L. (2015) Discovery of microbial natural products by activation of silent biosynthetic gene clusters. Nat Rev Micro 13, 509-523

90 Owen, J.G., et al. (2015) Multiplexed metagenome mining using short DNA sequence tags facilitates targeted discovery of epoxyketone proteasome inhibitors. Proceedings of the National Academy of Sciences 112, 4221-4226 
91 Charlop-Powers, Z., et al. (2014) Metagenomic small molecule discovery methods. Curr. Opin. Microbiol. 19, 70-75

92 Kang, H.-S. and Brady, S.F. (2013) Arimetamycin A: Improving Clinically Relevant Families of Natural Products through Sequence-Guided Screening of Soil Metagenomes. Angew. Chem. Int. Ed. 52, $11063-11067$

93 Penesyan, A., et al. (2015) Antibiotic Discovery: Combatting Bacterial Resistance in Cells and in Biofilm Communities. Molecules 20, 5286-5298

94 Fletcher, M.H., et al. (2014) Draining the moat: disrupting bacterial biofilms with natural products. Tetrahedron 70, 6373-6383

95 Piazza, V., et al. (2014) Antifouling Activity of Synthetic Alkylpyridinium Polymers Using the Barnacle Model. Mar. Drugs 12, 1959-1976

96 Friedman, M. (2015) Antibiotic-Resistant Bacteria: Prevalence in Food and Inactivation by Food-Compatible Compounds and Plant Extracts. J. Agric. Food Chem. 63, 3805-3822 97 Vergis, J., et al. (2015) Essential Oils as Natural Food Antimicrobial Agents: A Review. Crit. Rev. Food Sci. Nutr. 55, 1320-1323

98 Babu, K.M. and Ravindra, K.B. (2015) Bioactive antimicrobial agents for finishing of textiles for health care products. J. Text. Inst. 106, 706-717

99 Cheng, L., et al. (2015) Natural Products and Caries Prevention. Caries Research 49, 38-45 100 Hotwani, K., et al. (2014) Phytodentistry: use of medicinal plants. J. Complementary Integr. Med. 11, 233-251

101 Ling, L.L., et al. (2015) A new antibiotic kills pathogens without detectable resistance. Nature 517, 455-459

102 Jad, Y.E., et al. (2015) Synthesis and Biological Evaluation of a Teixobactin Analogue.

Organic Letters 17, 6182-6185

103 Son, S., et al. (2015) Ulleungamides A and B, Modified $\alpha, \beta$-Dehydropipecolic Acid Containing Cyclic Depsipeptides from Streptomyces sp. KCB13F003. Org. Lett. 17, 4046-4049

104 Hassan, H.M., et al. (2015) Salinamide F, new depsipeptide antibiotic and inhibitor of bacterial RNA polymerase from a marine-derived Streptomyces sp. J. Antibiot. 68, 206-209

105 Zaher, A.M., et al. (2015) A new enniatin antibiotic from the endophyte Fusarium tricinctum Corda. J. Antibiot. 68, 197-200

106 Essig, A., et al. (2014) Copsin, a Novel Peptide-based Fungal Antibiotic Interfering with the Peptidoglycan Synthesis. J. Biol. Chem. 289, 34953-34964

107 Cociancich, S., et al. (2015) The gyrase inhibitor albicidin consists of p-aminobenzoic acids and cyanoalanine. Nat Chem Biol 11, 195-197

108 Baumann, S., et al. (2014) Cystobactamids: Myxobacterial Topoisomerase Inhibitors Exhibiting Potent Antibacterial Activity. 53, 14605-14609

109 Mo, X., et al. (2014) Naturally occurring tetramic acid products: isolation, structure elucidation and biological activity. RSC Advances 4, 50566-50593

110 Halecker, S., et al. (2014) Hymenosetin, a 3-decalinoyltetramic acid antibiotic from cultures of the ash dieback pathogen, Hymenoscyphus pseudoalbidus. Phytochemistry 100, 86-91

111 Kauhl, U., et al. (2016) Total Synthesis of (-)-Hymenosetin. J. Org. Chem. 81, 215-228 112 Singh, S.B., et al. (2015) Kibdelomycin is a bactericidal broad-spectrum aerobic antibacterial agent. Antimicrob. Agents Chemother. 59, 3474-3481

113 Miesel, L., et al. (2014) Kibdelomycin Is a Potent and Selective Agent against Toxigenic Clostridium difficile. Antimicrob. Agents Chemother. 58, 2387-2392

114 Singh, S.B., et al. (2012) Kibdelomycin A, a congener of kibdelomycin, derivatives and their antibacterial activities. Biorg. Med. Chem. Lett. 22, 7127-7130

115 Hoshino, S., et al. (2015) Dietziamides, novel tetramic acid dimers from Dietzia timorensis MZ-3 with antioxidative activity. Bioorg. Med. Chem. Lett. 25, 3953-3955

116 Guo, W., et al. (2015) Penicyclones A-E, Antibacterial Polyketides from the Deep-Sea-

Derived Fungus Penicillium sp. F23-2. J. Nat. Prod. 78, 2699-2703 
117 Yoshino, M., et al. (2012) Organocatalytic asymmetric syntheses of inthomycins A, B and C. Org. Biomol. Chem. 10, 8164-8174

118 Bulger, P.G., et al. (2003) A multicomponent coupling strategy suitable for the synthesis of the triene component of the oxazolomycin antibiotics. Org. Biomol. Chem. 1, 3726-3737

119 Bilyk, O., et al. (2016) New Simocyclinones: Surprising Evolutionary and Biosynthetic Insights. ACS Chem. Biol. 11, 241-250

120 Ishihara, J. and Hatakeyama, S. (2014) Total Synthesis of Oxazolomycins. Chem. Rec. 14, 663677

121 Klochko, V.V., et al. (2016) Prospects of a new antistaphylococcal drug batumin revealed bymolecular docking and analysis of the complete genome sequence ofthe batumin-producer Pseudomonas batumici UCM B-321. Int. J. Antimicrob. Agents 47, 56-71

122 Tripathi, A., et al. (2014) Correction to "Baulamycins A and B, Broad-Spectrum Antibiotics Identified as Inhibitors of Siderophore Biosynthesis in Staphylococcus aureus and Bacillus anthracis”. J. Am Chem. Soc. 136, 10541-10541

123 Tripathi, A., et al. (2014) Baulamycins A and B, Broad-Spectrum Antibiotics Identified as Inhibitors of Siderophore Biosynthesis in Staphylococcus aureus and Bacillus anthracis. J. Am. Chem. Soc. 136, 1579-1586

124 Farooq, S., et al. (2014) Artonin I inhibits multidrug resistance in Staphylococcus aureus and potentiates the action of inactive antibiotics in vitro. J. Appl. Microbiol. 117, 996-1011

125 Huang, J.-K., et al. (2015) Studies on Antibiotics Active against Resistant Bacteria. Total Synthesis of MRSA-Active Tetarimycin A and Its Analogues. Org. Lett. 17, 4248-4251

126 Shang, Z., et al. (2015) Viridicatumtoxins: Expanding on a Rare Tetracycline Antibiotic Scaffold. J. Org. Chem. 80, 12501-12508

127 Xia, X., et al. (2015) Barceloneic acid C, a new polyketide from an endophytic fungus Phoma sp. JS752 and its antibacterial activities. J Antibiot 68, 139-141

128 Barrett, T.N. and Barrett, A.G.M. (2014) Cascade Polyketide and Polyene Cyclizations:

Biomimetic Total Synthesis of Hongoquercin B. J. Am. Chem. Soc. 136, 17013-17015

129 Cushnie, T.P.T., et al. (2014) Alkaloids: An overview of their antibacterial, antibiotic-

enhancing and antivirulence activities. Int. J. Antimicrob. Agents 44, 377-386

130 Han, W.B., et al. (2014) Curvulamine, a New Antibacterial Alkaloid Incorporating Two

Undescribed Units from a Curvularia Species. Org. Lett. 16, 5366-5369

131 Rodriguez, R.A., et al. (2014) Axinellamines as Broad-Spectrum Antibacterial Agents:

Scalable Synthesis and Biology. J. Am. Chem. Soc. 136, 15403-15413

132 Coppo, E. and Marchese, A. (2014) Antibacterial Activity of Polyphenols. Curr. Pharm.

Biotechnol. 15, 380-390

133 Singh, P., et al. (2014) Recent developments in biological activities of chalcones: A mini review. Eur. J. Med. Chem. 85, 758-777

134 Cochrane, S.A., et al. (2015) Synthesis of Tridecaptin-Antibiotic Conjugates with in Vivo Activity against Gram-Negative Bacteria. J. Med. Chem. 58, 9779-9785

135 Betts, J.W., et al. (2015) In Vitro Activity of Epigallocatechin Gallate and Quercetin Alone and in Combination versus Clinical Isolates of Methicillin-Resistant Staphylococcus aureus. Org. Lett. 78, 2145-2148

136 Santhosh, R.S. and Suriyanarayanan, B. (2014) Plants: A Source for New Antimycobacterial Drugs. Planta Medica 80, 9-21

137 Mahajan, A., et al. (2014) Synthesis and medicinal chemistry of selected antitubercular natural products and natural product derivatives. Rsc Advances 4, 15180-15215

138 Sansinenea, E. and Ortiz, A. (2014) Antitubercular Natural Terpenoids: Recent Developments and Syntheses. Current Organic Synthesis 11, 545-591

139 Keni, R.S., et al. (2015) Recent progress in the drug development of coumarin derivatives as potent antituberculosis agents. Eur. J. Med. Chem. 100, 257-269

140 Sundarrajan, S., et al. (2015) Computational evaluation of phytocompounds for combating drug resistant tuberculosis by multi-targeted therapy. J. Mol. Model. 21, 247 
141 Yokokawa, F. (2014) Recent Progress on the Development of Novel Antitubercular Agents from Whole-Cell Screening Hits. Journal of Synthetic Organic Chemistry Japan 72, 1239-1249 142 Aviles, E., et al. (2013) Two Rare-Class Tricyclic Diterpenes with Antitubercular Activity from the Caribbean Sponge Svenzea flava. Application of Vibrational Circular Dichroism Spectroscopy for Determining Absolute Configuration. J. Org. Chem. 78, 11294-11301

143 Chen, C., et al. (2013) Three antimycobacterial metabolites identified from a marine-derived Streptomyces sp MS100061. Appl. Microbiol. Biotechnol. 97, 3885-3892

144 Kumar, M.M.K., et al. (2014) Denigrins A-C: new antitubercular 3,4-diarylpyrrole alkaloids from Dendrilla nigra. Natural Product Research 28, 888-894

145 Wijeratne, E.M.K., et al. (2013) Phomapyrrolidones A-C, Antitubercular Alkaloids from the Endophytic Fungus Phoma sp. NRRL 46751. J. Nat. Prod. 76, 1860-1865

146 Canche Chay, C.I., et al. (2014) Synthesis and anti-tuberculosis activity of the marine natural product caulerpin and its analogues. Mar. Drugs 12, 1757-1772

147 Arai, M., et al. (2014) Identification of the Target Protein of Agelasine D, a Marine Sponge Diterpene Alkaloid, as an Anti-dormant Mycobacterial Substance. Chembiochem 15, 117-123 148 Liu, M., et al. (2014) Endophytic Streptomyces sp Y3111 from traditional Chinese medicine produced antitubercular pluramycins. Appl. Microbiol. Biotechnol. 98, 1077-1085

149 Chen, S., et al. (2014) Three New Phenylpropanoids from the Roots of Piper taiwanense and Their Inhibitory Activities on Platelet Aggregation and Mycobacterium tuberculosis. Chemistry \& Biodiversity 11, 792-799

150 Zhou, H., et al. (2015) Anti-Mycobacterium tuberculosis Active Metabolites from an Endophytic Streptomyces sp YIM65484. Records of Natural Products 9, 196-200

151 Ganihigama, D.U., et al. (2015) Antimycobacterial activity of natural products and synthetic agents: Pyrrolodiquinolines and vermelhotin as anti-tubercular leads against clinical multidrug resistant isolates of Mycobacterium tuberculosis. Eur. J. Med. Chem. 89, 1-12

152 Dey, D., et al. (2014) Antitubercular and Antibacterial Activity of Quinonoid Natural Products Against Multi-Drug Resistant Clinical Isolates. Phytotherapy Research 28, 1014-1021

153 Aviles, E., et al. (2015) Synthesis and preliminary biological evaluation of a small library of hybrid compounds based on Ugi isocyanide multicomponent reactions with a marine natural product scaffold. Bioorg. Med. Chem. Lett. 25, 5339-5343

154 Shen, L., et al. (2013) Syntheses and evaluation of macrocyclic engelhardione analogs as antitubercular and antibacterial agents. J. Antibiot. 66, 319-325

$155 \mathrm{Xu}, \mathrm{S}$. , et al. (2014) Synthesis and antimycobacterial evaluation of natural oridonin and its enmein-type derivatives. Fitoterapia 99, 300-306

$156 \mathrm{Xu}, \mathrm{S} .$, et al. (2014) Design, synthesis and antimycobacterial activity evaluation of natural oridonin derivatives. Bioorg. Med. Chem. Lett. 24, 2811-2814

157 Holzgrabe, U. (2015) New Griselimycins for Treatment of Tuberculosis. Chemistry \& Biology 22, 981-982

158 Bhatti, H.N., et al. (2014) Biotransformation of monoterpenoids and their antimicrobial activities. Phytomedicine 21, 1597-1626

159 Matsuzawa, A., et al. (2014) Direct Aldol Strategy in Enantioselective Total Synthesis of Thuggacin B. Chem. - Eur. J. 20, 68-71

$160 \mathrm{Li}, \mathrm{X} .-\mathrm{W}$., et al. (2013) Bio-Inspired Formal Synthesis of Hirsutellones A-C Featuring an Electrophilic Cyclization Triggered by Remote Lewis Acid-Activation. Chem. - Eur. J. 19, 1638916393

161 Nakamura, H., et al. (2015) Total Synthesis of (-)-Caprazamycin A. Angew. Chem. Int. Ed. 54, 3136-3139

162 Wang, Y., et al. (2013) Improved Synthesis of Capuramycin and Its Analogues. Chem. - Eur. J. 19, 13847-13858

163 Danishefsky, S. (2010) On the potential of natural products in the discovery of pharma leads: A case for reassessment. Nat. Prod. Rep. 27, 1114-1116 
164 Butler, M.S. and Buss, A.D. (2006) Natural products - The future scaffolds for novel antibiotics? Biochem. Pharmacol. 71, 919-929

165 Nussbaum, F.v., et al. (2006) Antibacterial Natural Products in Medicinal Chemistry-Exodus or Revival? Angew. Chem. Int. Ed. 45, 5072-5129

166 Clardy, J., et al. (2006) New antibiotics from bacterial natural products. Nat Biotech 24, 15411550

167 Butler, M.S. (2004) The Role of Natural Product Chemistry in Drug Discovery. J. Nat. Prod. 67, 2141-2153

168 Patridge, E., et al. (2016) An analysis of FDA-approved drugs:natural products and their derivatives. Drug Discovery Today 21, 204-207

169 Dwivedi, G.R., et al. (2014) 4-Hydroxy- a- Tetralone and its Derivative as Drug Resistance Reversal Agents in Multi Drug Resistant Escherichia coli. Chem. Biol. Drug Des. 83, 482-492 170 Brown, D. (2015) Antibiotic resistance breakers: can repurposed drugs fill the antibiotic discovery void? Nat. Rev. Drug Discovery 14, 821-832

171 Velkov, T., et al. (2014) Teaching 'Old’ Polymyxins New Tricks: New-Generation Lipopeptides Targeting Gram-Negative 'Superbugs'. ACS Chemical Biology 9, 1172-1177 172 Velkov, T., et al. (2016) A Novel Chemical Biology Approach for Mapping of Polymyxin Lipopeptide Antibody Binding Epitopes. ACS Infectious Diseases

173 Schobert, R. and Schlenk, A. (2008) Tetramic and tetronic acids: An update on new derivatives and biological aspects. Bioorg. Med. Chem. 16, 4203-4221

174 Royles, B.J.L. (1995) Naturally-Occurring Tetramic Acids - Structure, Isolation, And Synthesis. Chem. Rev. 95, 1981-2001

175 Jeong, Y.-C. and Moloney, M.G. (2015) Antibacterial barbituric acid analogues inspired from natural 3-acyltetramic acids; Synthesis, tautomerism and structure and physicochemical propertyantibacterial activity relationships. Molecules 20, 3582-3627

176 Jeong, Y.-C., et al. (2013) Natural product inspired antibacterial tetramic acid libraries with dual enzyme target activity. Chem. Sci. 4, 1008-1015

177 Jeong, Y.-C. and Moloney, M.G. (2009) Tetramic acids as bioactive templates: synthesis, tautomeric and antibacterial behaviour. Synlett., 2487-2491

178 Baell, J.B. (2016) Feeling Nature’s PAINS: Natural Products, Natural Product Drugs, and Pan Assay Interference Compounds (PAINS). J. Nat. Prod. 79, 616-628

179 Bisson, J., et al. (2016) Can Invalid Bioactives Undermine Natural Product-Based Drug Discovery? J. Med. Chem. 59, 1671-1690

180 Blaskovich, M.A.T., et al. (2015) Helping Chemists Discover New Antibiotics. ACS Infect. Dis. 1, 285-287

181 Cooper, M.A. (2015) A community-based approach to new antibiotic discovery. Nat. Rev. Drug Discovery 14, 587-588

182 Johnston, C.W., et al. (2016) Assembly and clustering of natural antibiotics guides target identification. Nat Chem Biol 12, 233-239

183 Warp drive. http://www.warpdrivebio.com 